\title{
La place d'Alcuin dans la rédaction épigraphique carolingienne
}

\section{Cécile Treffort}

\section{(C) OpenEdition}

1 Journals

\section{Édition électronique}

URL : http://journals.openedition.org/abpo/1258

DOI : 10.4000/abpo. 1258

ISBN : 978-2-7535-1495-9

ISSN : 2108-6443

Éditeur

Presses universitaires de Rennes

Édition imprimée

Date de publication : 20 septembre 2004

Pagination : 353-369

ISBN : 978-2-7535-0053-2

ISSN : 0399-0826

\section{Référence électronique}

Cécile Treffort, "La place d'Alcuin dans la rédaction épigraphique carolingienne ", Annales de Bretagne et des Pays de l'Ouest [En ligne], 111-3 | 2004, mis en ligne le 20 septembre 2006, consulté le 01 mai 2019. URL : http://journals.openedition.org/abpo/1258; DOI : 10.4000/abpo.1258 


\title{
La place d'Alcuin dans la rédaction épigraphique carolingienne
}

\author{
Cécile TREFFORT \\ Maître de conférences en histoire médiévale à l'Université de Poitiers, CESCM
}

Parmi les différents aspects de la culture carolingienne, le domaine des inscriptions, souvent considéré comme du ressort exclusif des épigraphistes ou - éventuellement - des archéologues, a longtemps été négligé par les historiens ou les littéraires pour qui l'écrit n'a que deux dimensions. Pourtant, les textes des inscriptions, souvent monumentaux, sculptés dans la pierre ou peints sur les murs des édifices, s'imposaient à la vue de tous, de manière durable ${ }^{1}$. Devenant en quelque sorte des textes publics, leur portée est différente de celle de manuscrits confinés dans une bibliothèque monastique et des supports variés peuvent parfois assurer en parallèle la diffusion d'un même texte dans des optiques diverses, voire complémentaires.

Loin d'être un genre mineur, la rédaction de carmina épigraphiques, à l'époque carolingienne, est le fait de prestigieux auteurs, parmi lesquels Alcuin. Lui-même s'est inspiré d'inscriptions antérieures - connues par des copies manuscrites ou par la tradition lapidaire - pour ses propres compositions. Il a rédigé des textes pour plusieurs établissements religieux, à la demande de ses proches ${ }^{2}$, ainsi que deux épitaphes célébrissimes dans le monde carolingien : celle du pape Hadrien $\mathrm{I}^{\text {er }} 3$ et la sienne ${ }^{4}$. La diffusion de ces deux œuvres, grâce à leur copie dans divers manuscrits, s'inscrit dans un empire qui se nourrit de la réforme de la langue et de l'écriture;

1. Pour une définition des inscriptions fondée non sur la nature du support mais sur la finalité des textes épigraphiques, voir R. FAVREAu, Epigraphie médiévale, p. 5.

2. Liste dans M.-H. Jullien et F. Perelman, Clavis..., aux entrées "Epitaphia " et "Inscriptiones".

3. Éd. MGH Poet. 1, p. 36. Plusieurs études majeures ont été consacrées à cette inscription, en particulier (par ordre alphabétique) : I. B. DE RossI, « L'inscription du tombeau d'Hadrien I... »; J. RAMACKERS, « Die Werkstattheimat der Grabplatte... »; S. SCHOLZ, « Karl der Große und das "Epitaphium Hadriani”... »; L. WALLACH, " Alcuin's Epitaph of Hadrian I... ».

4. Éd. MGH Poet. 1, p. 350-351. Étudiée en particulier par L. WALLACH, « The Epitaph of Alcuin... ". 
ce n'est sans doute pas un hasard si ces pièces servent par la suite de modèle, littéraire ou épigraphique.

L'influence d'Alcuin en matière épigraphique se mesure à la réception de ses textes par exemple chez Raban Maur, son disciple, ou chez d'autres auteurs moins directement liés à sa personne. Surtout, elle est sensible dans l'utilisation de ses œuvres poétiques non épigraphiques dans la composition d'inscriptions lapidaires. Le caractère à la fois matériel et littéraire des inscriptions rend souvent complexe la compréhension de l'origine et de la circulation des formules dans l'empire; une telle recherche, si elle n'est qu'esquissée au cours de cette présentation, s'avère toutefois nécessaire pour explorer des voies nouvelles dans l'histoire culturelle, mêlant culture matérielle et culture écrite, trop souvent séparées ${ }^{5}$.

\section{Alcuin et les traditions épigraphiques antérieures}

On sait depuis longtemps qu'Alcuin a utilisé, pour ses compositions, des textes antérieurs, qu'ils relèvent de l'Antiquité païenne (Ovide, Virgile...) ou chrétienne (Paulin de Nole ou Venance Fortunat par exemple). Il suffit, pour s'en convaincre, de lire les notes en bas de page des Monumenta Germaniae Historica qui représentent une faible partie des sources mises à contribution. L'arrivée des CD-Rom, qui complètent - sans les remplacer - les ouvrages traditionnels, aide considérablement le chercheur privé de la vaste culture d'un lettré carolingien et permet de mieux mesurer la multiplicité des emprunts et la virtuosité de certains centons. Cependant, ils ne permettent pas d'appréhender les liens entre Alcuin et les traditions épigraphiques, les éditions d'inscriptions étant dispersées et encore loin d'être exhaustives pour l'ensemble de l'empire. Remarqués dès 1856 par Edmond Le Blant ${ }^{6}$, ces rapports n'ont guère été étudiés jusqu'à présent de manière systématique et il reste impossible, actuellement, d'en mesurer l'ampleur et d'en dessiner clairement les contours. Souvent, il est difficile de choisir entre une simple parenté et des liens plus précis de dépendance, de réception, de citation ou de réminiscence, lorsqu'on ne peut préciser le processus exact de transmission - directe ou indirecte des formules. Quelques exemples significatifs permettent toutefois d'entrevoir une réalité souvent ignorée des chercheurs.

Alcuin utilise par exemple des textes rédigés par le pape Damase, dont il semble avoir eu connaissance par des sylloges épigraphiques ${ }^{7}$ en

5. Nous laisserons à plus compétent(e) le soin de déterminer la nature exacte de la mutation paléographique qui affecte les inscriptions lapidaires au début du $\mathrm{x}^{\mathrm{e}}$ siècle. Voir en particulier les pistes lancées par F. DE RuBEIS, "Sillogi epigrafiche... ". Je remercie l'auteur de m'avoir transmis son texte avant publication.

6. E. LE BLANT, Inscriptions chrétiennes de la Gaule..., t. I, préface, p. cXXXIII.

7. Le travail fondamental sur ces sylloges reste la publication de I. B. DE RossI, Inscriptiones christianae..., vol. II, 1; on peut également se reporter à la notice de H. LECLERCQ, "Inscriptions (Histoire des recueil d') ». Les travaux plus récents, mais plus ponctuels, seront détaillés dans les notes suivantes. 
Angleterre ou sur le continent. On peut citer l'exemple du vers Iustitiae cultor, présent uniquement dans la sylloge dite de Tours, qu'Alcuin a utilisé plusieurs fois ${ }^{8}$, la transmettant également à Raban Maur :

Sylloge de Tours ${ }^{9}, 27,9$.

Iustitiae cultor vitae servator honestae

Alcuin, Poème sur York, v. 134

Cui datur antistes vitae servator honestae

Nomine Paulinus.../Qui fuit.../

Iustitiae cultor, verus pietatis amator ${ }^{10}$.

Cette dernière phrase se retrouve dans trois autres poèmes :

Alc. Carm. 15, v. 5 (à Léon) : Iustitiae cultor verae et pietatis amator

Alc. Carm. 17, v. 15 (à Paulin) : Iustitiae cultor sacrae et pietatis amator

Alc. Carm. 43, v. 5 (au pape Léon) : Iustitiae cultor sanctae et pietatis amator

Ce dernier vers est repris par Raban Maur

Raban Maur, Carm. 13, 7 : Iustitiae cultor sanctae et pietatis amator

Alcuin puise également à des épitaphes pontificales ${ }^{11}$ qui ont pu être transmises par différentes sylloges. L'inscription funéraire du pape Jean VII ne figure, parmi les sylloges carolingiennes connues, que dans celle de Cambridge ${ }^{12}$, qu'Alcuin semble avoir utilisée avant de se rendre sur le continent ${ }^{13}$ :

Épitaphe du pape Jean VII (mort en 707)

Non parcens opibus pretiosum quicquid habebat

In tua distribuit munera sancta parens.

Alcuin, Poème sur York, v. 284

O pietas o celsa fides nam quidquid habebat

Prodigus in Domini gazarum sparsit honorem.

Alc. Carm. 14, v. 3 (inscription pour Saint-Amand)

Non parcens opibus miseris nam quidquid habebat

Sparserat et Christi compsit sacra templa sacerdos.

Alc. Carm. 66, 2, v. 8 (inscription pour Laon, 798)

Non parcens propriis opibus nam quidquid habebat

Distribuit larga mente in donaria Christi.

Angilbert, Carm. 4, v. 5

Non parcens opibus propriis nam quidquid habebat

Ecclesia larga mente iam pietate dedit.

On peut ainsi multiplier les exemples : in arce poli, dont on trouve vingtdeux occurrences chez Alcuin, pourrait provenir de l'épitaphe du roi

8. Tradition évoquée partiellement par L. WALLACH, « The Epitaph of Alcuin... », p. 371 puis, de manière plus précise, dans " Alcuin's Epitaph of Hadrian I... ", p. 142-143.

9. I. B. DE Rossi, Inscriptiones christianae ..., vol. II, $1, \mathrm{n}^{\circ} \mathrm{VI}, 27,9$, p. 67.

10. Cf. L. WALLACH, "The Epitaph of Alcuin... ", p. 371.

11. L'utilisation ou l'imitation des inscriptions des plus anciennes basiliques romaines dans l'épigraphie médiévale a déjà été remarquée par R. FAVREAU, "La mémoire du passé..." ", p. 944-948.

12. La sylloge dite de Cambridge, reconstituée d'après un manuscrit du Liber pontificalis du XII ${ }^{\mathrm{e}}$ siècle conservé à Cambridge et intégrant dans les notices pontificales des textes épigraphiques, a été publiée pour la première fois en 1910 par W. LEVISON, puis par L. DuCHESNE, " Le recueil épigraphique de Cambridge " et, en dernier lieu, par A. SILVAGNI, "La silloge epigrafica di Cambridge".

13. Tradition développée par L. WALLACH, « The Epitaph of Alcuin... ", p. 372. 
Cedwal visible dans la basilique Saint-Pierre et rapportée dans diverses sylloges ${ }^{14}$, à moins qu'elle n'ait été empruntée à une autre inscription, transmise par la sylloge de Tours et qui se voyait dans la basilique des saints Cosme et Damien ${ }^{15}$; de même, tecta sacrata, qu'Alcuin utilise cinq fois, se retrouve dans la sylloge palatine ${ }^{16}$; l'expression virgo sacrata Deo, utilisée dans deux inscriptions d'Alcuin, a son équivalent dans la série milanaise d'une des sylloges de Lorsch ${ }^{17}$. Regna beata petit, qu'Alcuin utilise cinq fois sous cette forme précise et plusieurs autres fois avec diverses variantes, est présent dans une inscription de Vercelli, rapportée également par une sylloge ${ }^{18}$. Il faudrait pouvoir rendre systématique cette recherche afin d'identifier les collections épigraphiques qu'Alcuin avait à sa disposition aux différentes périodes de sa vie et déterminer la proportion des emprunts aux textes épigraphiques par rapport aux autres sources. Il faudrait sans doute également étendre l'enquête à d'autres auteurs qui, comme Angilbert de Saint-Riquier ${ }^{19}$ ou, plus tard, Flodoard de Reims ${ }^{20}$, ont également puisé dans les textes des inscriptions romaines transmises par les sylloges pour leurs compositions métriques. Il suffira de citer ici l'exemple bien connu de la dédicace de l'évangile de Godelscalc à Charles le Chauve ${ }^{21}$, texte versifié dont les vers 6 et 9 sont issus de l'épitaphe du pape Félix IV et le vers 8 de celle de Boniface III $^{22}$. Les vers 8 et 9 de ce dernier texte ont d'ailleurs été utilisés dans l'épitaphe d'Haistulphe de Mayence (mort en 825) rédigée par Raban Maur ${ }^{23}$.

Qu'Alcuin s'inspire de la tradition épigraphique romaine, en particulier pontificale, pour ses propres compositions, n'a en soit rien d'étonnant. Rome est le point de mire de l'Église telle que la rêvent les Carolingiens.

14. I. B. DE RossI, Inscriptiones christianae..., vol. II, 1, $\mathrm{n}^{\circ} \mathrm{VI}, 40$, p. 70 (sylloge de Tours), $\mathrm{n}^{\circ}$ VII, 4, p. 79 (sylloge de Saint-Riquier), $\mathrm{n}^{\circ}$ VIII, 72, p. 111-112 (4 ${ }^{\mathrm{e}}$ sylloge de Lorsch) notamment et p. 288.

15. I. B. DE RossI, Inscriptiones christianae..., vol. II, 1, $\mathrm{n}^{\circ} \mathrm{VI}, 41,6$, p. 71 : Munus ut etheria vivat in arce poli; Alc. Carm. 65 , 1a, 20 : Ut felix tecum vivat in arce poli. Éd. MGH Poet. 1 , p. 285.

16. I. B. DE RossI, Inscriptiones christianae..., vol. II, 1, $\mathrm{n}^{\circ}$ VIII, 55, p. 107. La composition de ce manuscrit (Rome, Biblioteca apostolica vaticana, ms. Palat. lat. 833) de même que la distribution et la datation des différentes collections épigraphiques qui y sont copiées ont été réétudiées récemment par C. VIRCILLO FRANKLIN, "The epigraphic sylloge..." .

17. I. B. DE RossI, Inscriptiones christianae..., vol. II, 1, n XVI, 4, p. 162.

18. I. B. DE Rossi, Inscriptiones christianae..., vol. II, 1, n I, p. 314.

19. Éd. MGH SS, XV, 1, p. 177-179, en lien avec la Sylloge III de Lorsch, éd. I. B. DE RossI, Inscriptiones christianae..., vol. II, $1, \mathrm{n}^{\circ} \mathrm{XVI}$.

20. De Christi triumphis apud Italiam libri quatuordecim (PL 135, col. 823-831); R. FAVREAU, "La mémoire du passé... ", p. 948 a ainsi remarqué des emprunts aux épitaphes pontificales pour Hadrien II, Jean VIII, Marin I ${ }^{\text {er }}$, Etienne VI, Jean IX, Benoît IV, Serge III, Anastase III.

21. Paris, Bibliothèque nationale de France, ms. nouv. acq. lat. 1203. Éd. MGH Poet. 1, p. 94 : v. 6 : Praelatus multis humili pietate superbus; v. 8 : Iustitiae custos rectus verusque fidelis; v. 9 : Pauperibus largus miseris solacia praestans. Texte analysé par L. WALLACH, "Alcuin's Epitaph of Hadrian I... ", p. 143-144.

22. I. B. DE RossI, Inscriptiones christianae..., vol. II, $1, \mathrm{n}^{\circ} \mathrm{V}, 18$, p. 57 et $\mathrm{n}^{\circ} \mathrm{XI}, 3$, p. 126 (Félix IV), ${ }^{\circ}$ XI, 1, p. 126 et $\mathrm{n}^{\circ}$ XII, 31, p. 141 (Boniface III).

23. Raban Maur, Carm. 84, vers 8-9. Éd. MGH Poet. 2, p. 237. 
Les liens entre ses textes et les inscriptions romaines sont tellement étroits qu'ils circulent ensemble dans les manuscrits, ce qui pose parfois des problèmes d'attribution particulièrement complexe ${ }^{24}$.

Plusieurs des textes attribués à Alcuin par Ernst Dümmler (série 114) figurent ainsi dans la sylloge de Milred, évêque de Worcester mort en 775, connue indirectement par des extraits copiés au $\mathrm{x}^{\mathrm{e}}$ siècle et par son utilisation dans le liber pontificalis de Guillaume de Malmesbury ${ }^{25}$. Les cinq premiers vers de l'épitaphe du pape Jean II mort en 535 (Mente pia vivens Christi nutritus in aula) ont été intégrés dans une série alcuinienne copiée au milieu du $\mathrm{IX}^{\mathrm{e}}$ siècle dans un manuscrit bavarois aujourd'hui conservé à Munich ${ }^{26}$ et publiés sous l'autorité d'Alcuin par Ernst Dümmler ${ }^{27}$. Dans un des manuscrits du Vatican, l'épitaphe d'Alcuin suit immédiatement celle que Damase a rédigée pour lui-même ${ }^{28}$. Dans un manuscrit de Valenciennes comprenant les carmina d'Alcuin, on trouve également les épitaphes des saints papes Boniface et Grégoire, ainsi que celle de Monique, mère de saint Augustin ${ }^{29}$.

Toutefois, les emprunts aux textes épigraphiques ne sont pas tributaires uniquement de la tradition manuscrite des inscriptions. Alcuin a pu avoir connaissance également d'autres textes, de nature lapidaire, plus difficiles à appréhender mais aussi riches d'enseignement. Dans une inscription rédigée pour Saint-Hilaire de Poitiers ${ }^{30}$, Alcuin utilise une expression originale, quos tulit una dies, qu'on retrouve sur une pierre aujourd'hui conservée à Naples et qui provient de Rome ${ }^{31}$. Sans forcément établir de lien direct entre les deux (même si Alcuin a pu voir cette inscription lors d'un de ses passages dans la cité romaine), on peut garder à l'esprit qu'une formule épigraphique pour l'instant non répertoriée parmi les carmina manuscrits a pu être utilisée ici ${ }^{32}$.

Plus suggestive, on peut citer une petite inscription gravée sur ardoise, découverte au XIX ${ }^{\mathrm{e}}$ siècle à Vertou près de Nantes (figure 1) et conservée en cette ville, au musée Dobrée ${ }^{33}$. Fragmentaire, elle ne porte malheureu-

24. Déjà signalé par I. B. DE RossI, Inscriptiones christianae..., vol. II, 1, p. 281 (Tituli et epitaphia vetera mixta carminibus Alcuini).

25. P. Sims-WiLLIAMS, « Milred of Worcester's collection... ».

26. München, Staatsbibliothek, ms. Clm 19410, $\mathrm{f}^{\circ}$ 36. Cf. I. B. DE RossI, Inscriptiones christianae..., vol. II, $1, \mathrm{n}^{\circ}$ XXVII, 7, p. 286. D. SCHALLER, "Bemerkungen zur InschriftenSylloge... ", p. 17, suggère que les œuvres d'Alcuin ont pu arriver en Bavière par l'intermédiaire d'Arno de Salzbourg.

27. Éd. MGH Poet. 1, p. 268, Carm. 55, 10.

28. Roma, Biblioteca apostolica vaticana, ms. Reg. lat. $1578, \mathrm{f}^{\circ} 26 \mathrm{r}^{\circ}-\mathrm{v}^{\circ}$.

29. Valenciennes, Bibliothèque municipale, ms. 387. Cité par I. B. DE Rossı, Inscriptiones christianae..., vol. II, 1, p. 251.

30. Alc. Carm. 99, 14, 3. Éd. MGH Poet. 1, p. 326.

31. Éd. E. Buchler, Carmina Epigraphica Latina, vol. II, nº 1159, 2, p. 536.

32. Même si l'on ne peut exclure une influence de poètes latins comme Ovide (abstulit una dies) ou Fortunat (tulit una dies) par exemple. Cf. O. Schumann, Lateinisches HexameterLexikon..., vol. 5, p. 701.

33. Nantes, Musée Dobrée, $\mathrm{n}^{\circ}$ inv. 900.7.1. Publiée pour la première fois par 
sement ni le nom du défunt, ni la date. Toutefois, la comparaison paléographique avec des inscriptions régionales et précisément datées ${ }^{34}$ invite à placer la réalisation de cette plaque à l'extrême fin du VIII ${ }^{\mathrm{e}}$ siècle. Elle est donc légèrement antérieure ou tout au plus strictement contemporaine de la rédaction par Alcuin de sa propre épitaphe, nécessairement antérieure à mai 804 , date de sa mort. Culturellement, elle précède la réforme de l'écriture - dont Alcuin fut un des ardents promoteurs - et l'adoption de la capitale romaine qui remplaça la calligraphie anguleuse de la fin du $\mathrm{VIII}^{\mathrm{e}}$ siècle dans les premières décennies du IX ${ }^{\mathrm{e}}$ siècle. Or, le texte porté par cette petite plaque, vraisemblablement réalisée dans un atelier angevin, est le suivant :

\section{Obsecro ut nulla manus.' \\ violet pia iu[ra s]epulchri : \\ Donec p(er)so[net ange]lica.' \\ vox ab arche [poli :] \\ $[$ Hic] requiescit bo[ne memorie...}

Il s'agit d'un texte proche, dans sa formulation ${ }^{35}$, d'un distique de l'épitaphe d'Alcuin (Obsecro nulla manus violet pia iura sepulcri/Personet angelica donec ab arce tuba ${ }^{36}$ ) dont on retrouve des éléments dans les épitaphes de Dungal de Saint-Denis, mort en 827 (Oroque nemo mei violet pia iura sepulcri ${ }^{37}$ ) ou d'Adventius de Metz, mort en 875 (Obsecro per trinum Dominum contestor et unum/Mausolei septum nulla manus violet ${ }^{38}$ ).

La formule métrique de l'épitaphe d'Alcuin, à la postérité amplifiée par sa diffusion importante dans la tradition manuscrite, serait donc née d'une reprise d'un texte épigraphique antérieur, circulant sous forme lapidaire, orale ou, éventuellement, manuscrite même si on n'en a pour l'instant pas retrouvé trace.

Ch. Marionnaud, "Collections archéologiques... ", p. 337-340. Signalée également par R. FAVREAU, "La mémoire du passé... ", p. 971. Je profite de l'occasion pour remercier ici $\mathrm{M}^{\text {me }}$ Santrot, conservateur, de m'avoir permis l'accès aux réserves du musée et sa collaboratrice, Séverine Chaudrier, pour son accueil et son aide.

34. Les comparaisons les plus pertinentes sont vraisemblablement à faire avec deux inscriptions angevines, sculptées comme celle de Vertou sur ardoise, datées respectivement de 771 (Erdramnus) et de 784 (Autbertus), et conservées au Service départemental d'archéologie. Un dessin a été publié par D. PRIGENT, J.-Y. HunOT, dans La mort : voyage au pays des vivants..., p. 35. On y retrouve l'usage des enclavements et des conjonctions, une ponctuation logique par trois ou quatre points superposés, le dépassement des hastes verticales, la forme anguleuse de nombreuses lettres et les empattements triangulaires marqués. Je tiens à remercier ici Daniel Prigent, archéologue départemental du Maine-et-Loire pour son amitié et sa fidèle collaboration.

35. Je remercie à cette occasion mes collègues italiens Flavia de Rubeis et Francesco Stella pour l'analyse de ce texte qui semble être une imitation maladroite d'un modèle métrique.

36. MGH Poet. 1, p. 351, v. 19-20.

37. H. SiLVESTRE, " La véritable épitaphe... ", p. 257.

38. F. KRAUS, Die christlichen Inschriften ..., n ${ }^{\circ} 303-302$, p. 148-149. 
Figure 1

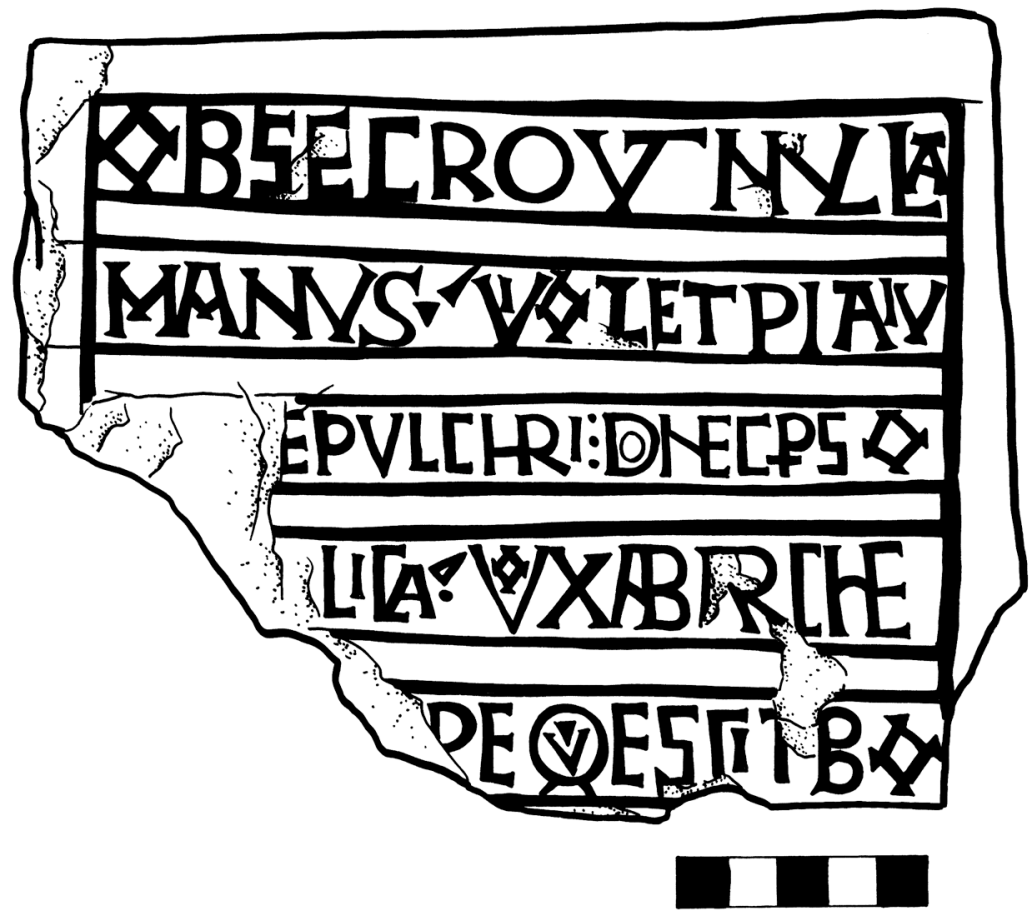

Alcuin apparaît finalement comme un passeur de mots qui utilise la forme épigraphique à la fois comme source d'inspiration et comme mode d'expression. En effet, Alcuin ne fait pas qu'emprunter à des carmina épigraphiques pour ses propres compositions; il rédige également des inscriptions dont le nombre (sinon l'ampleur) et la notoriété dépassent très largement celui de ses autres textes poétiques.

\section{Alcuin et l'écriture épigraphique}

L'écriture d'inscription est une manière d'élever le texte au rang de cadre de vie $^{39}$; Alcuin a privilégié cette forme pour diffuser son enseignement et celui des Écritures. Le caractère monumental et permanent des inscriptions peintes ou gravées dans l'environnement des frères ${ }^{40}$ a en effet permis à Alcuin d'amplifier l'œuvre d'édification, en créant un lien dynamique très particulier entre le texte et le fidèle. Dans le cas du manuscrit,

39. Pour une vue générale, on peut se reporter à l'article de F. STELLA, « Epigrafia letteraria e topografia...".

40. À San Vincenzo al Volturno, la préservation extraordinaire du monastère carolingien montre la réalité d'un tel environnement textuel monumental. Voir notamment J. MitCHELL, «Literacy Displayed... ». 
le lecteur a souhaité lire, il a ouvert le codex, il peut le refermer et l'écrit disparaît. Dans le cas de l'inscription, le texte est présent même sans lecteur; il s'impose à celui-ci, il l'interpelle, il crée par sa présence une logique spécifique de communication. Le texte est écrit pour un lieu particulier (hic, haec, hoc...) et il apostrophe le lecteur qui s'y rend; il l'aide à méditer sur les grandes vérités de la foi chrétienne et l'engage à prier.

Mis à part pour Saint-Martin de Tours, Alcuin a rédigé des inscriptions versifiées non pour les établissements relevant de son autorité ${ }^{41}$ mais pour ses proches. Dans un seul cas, on a l'assurance d'une commande, lorsqu'Alcuin écrit à Radon, abbé de Saint-Vaast d'Arras, et aux moines de l'abbaye : "Ainsi que me l'a demandé votre très chère charité et celle du seigneur abbé, nous avons dicté des vers pour chaque titulus des églises et pour chacun des autels ${ }^{42}$. "Dans les autres cas, on ignore si les destinataires de ces textes épigraphiques les avaient commandées ou s'ils les ont reçus en gage d'amitié. Parmi eux, on trouve ainsi plusieurs des correspondants favoris d'Alcuin, comme Ricbodo, évêque de Trêves et abbé de Lorsch, ou Arno, évêque de Salzbourg et abbé de Saint-Amand (Elnone). De même, saint Liudger, évêque de Münster, avait été son élève à York. Parfois, on ignore même la nature des rapports d'Alcuin avec le destinataire de l'inscription. C'est le cas d'Ato, abbé de Saint-Hilaire de Poitiers et de Nouaillé et devenu par la suite évêque de Saintes, qui doit peut-être la rédaction des vingt-huit inscriptions destinées à orner les deux abbayes après leur restauration (la dédicace de Nouaillé date de 799) ${ }^{43}$ à sa parenté avec Louis le Pieux ${ }^{44}$. Sans aller jusqu'à penser que ces inscriptions sont " la preuve péremptoire qu'Alcuin est venu à Poitiers ${ }^{45}$ ", la proximité de Tours ainsi que la présence à Chasseneuil (près de Poitiers) du palais du roi d'Aquitaine Louis le Pieux ne rend pas improbable un tel voyage. Les inscriptions seraient alors l'unique témoignage d'un lien personnel entre Ato et Alcuin.

Parmi les compositions épigraphiques d'Alcuin, seule l'épitaphe d'Hadrien I ${ }^{\text {er }}$ est encore conservée sous sa forme lapidaire; les autres ne nous sont connues que par leur copie dans des manuscrits, eux-mêmes parfois disparus. En ce qui concerne l'attribution des textes, les manuscrits donnent parfois des titres, mais pas toujours explicites; il faut alors

41. On ne connaît ainsi aucune inscription rédigée par Alcuin pour Flavigny, Saint-Josse ou Ferrières et l'attribution à Cormery du poème n ${ }^{\circ}$ 104, 6 est contestée. Cf. M.-H. JuLLIEN et F. Perelman, Clavis..., n ALC 61.105.

42. Alcuin, lettre aux moines de Saint-Vaast d'Arras (vers 796-804) : Sicut domni abbatis vestraque carissima caritas demandavit, versus per singulos titulos ecclesiarum et altaria singula dictavimus. Éd. MGH Ep. IV, 2, n 296, p. 454.

43. Éd. MGH Poet. 1, p. 323-327; voir aussi A. LARGEAULT, "Inscriptions métriques composées par Alcuin... " et Corpus des inscriptions de la France médiévale, I, p. 35-58 (avec traduction française).

44. Diplôme de Louis le Pieux : ...magnifico viro et parente nostro Atone diacono atque abbate... Cf. Ph. Depreux, Prosopographie de l'entourage de Louis le Pieux..., $\mathrm{n}^{\circ} 38$, p. 114-115.

45. A. LARGEAUlT, "Inscriptions métriques composées par Alcuin... », p. 225. 
croiser diverses informations telles que l'ordre dans lequel les poèmes sont cités dans le manuscrit, les personnages mentionnés dans le carmen, ou ce qu'on sait de la topographie des établissements supposés à l'époque d'Alcuin ${ }^{46}$.

Les inscriptions concernent essentiellement des églises et des autels; on compte notamment un poème de vingt hexamètres écrit vers 798 à la demande de Gerfrid, évêque de Laon, pour la cathédrale Sainte-Marie, un autre, en quatorze hexamètres, pour une église dédiée à saint Boniface par l'évêque de Münster Liudger, élève d'Alcuin à York, un poème en quatre distiques pour Trêves et l'évêque Ricbodo, deux poèmes pour Fleury-surLoire citant Magulfe, abbé mort en 794. Une des séries les plus importantes est celle qui comprend quatorze poèmes pour des autels, des oratoires, l'église Saint-Amand et la crypte Saint-Michel appartenant à l'abbaye de Saint-Amand (Elnone); ces poèmes, rédigés pour Arno, sont précédés, dans le manuscrit - perdu - de Saint-Bertin, de l'épitaphe de Gislebert, abbé de Saint-Amand et suivis de celle du saint. Il faut encore signaler une série de poèmes pour l'église Saint-Vaast d'Arras et divers autels, composés à l'occasion de sa reconstruction, après l'incendie de 793, par l'abbé Rado ${ }^{47}$, ainsi que deux poèmes destinés au monastère de Saint-Nabor de SaintAvold (près de Metz) citant l'abbé Vasco, qui succéda en 791 à Angilramne et termina le tombeau du saint commencé par ce dernier.

Certaines inscriptions accompagnent la vie conventuelle en prenant place non seulement dans l'église, mais également dans les espaces de vie ou de circulation du monastère. Ainsi, la série rédigée pour Saint-Martin de Tours comporte des textes pour l'école, le scriptorium, le dortoir, les latrines et un palais (à moins que celui-ci ne corresponde à Aix-la-Chapelle). Pour SaintHilaire de Poitiers, les inscriptions rédigées au temps de l'abbé Ato concernent quelques tombeaux, des oratoires, des autels et l'hôpital Saint-Pierrel'Houstault. Pour Nouaillé, reconstruite et consacrée par le même Ato en 799, Alcuin rédige également des inscriptions pour le réfectoire, le dortoir et l'hôtellerie chauffée. À Chelles, dont l'abbesse Gisèle, sœur de Charlemagne, est en correspondance avec Alcuin, les inscriptions concernent la bibliothèque, le réfectoire et sa cloche, le chœur de l'église et peut-être l'école.

On doit mettre à part la série d'inscriptions rédigées à la demande d'Arno, évêque de Salzbourg, à qui Alcuin avait déjà envoyé une série pour son abbaye de Saint-Amand. Il semblerait qu'Alcuin ait alors puisé dans ses archives et inséré, parmi quelques poèmes "nouveaux " (c'est-à-dire inconnus et peut-être composés pour l'occasion), les textes de plusieurs inscriptions déjà employées à Saint-Amand (oratoire Saint-Michel), Saint-Vaast d'Arras (autels Saint-Benoît-et-Sainte-Scholastique et Saint-Paul) et SaintHilaire de Poitiers (autel Saint-Jean et hôpital Saint-Pierre).

46. On doit d'ailleurs à ce titre saluer la publication récente de M.-H. JuLLIEN et F. Perelman, Clavis..., qui permet de démêler un peu l'écheveau.

47. L'article de A. LESTOCQUOY, « Notes sur l'épigraphie de l'abbaye... » complète l'édition des MGH. 
La reprise de ces textes s'insère dans la tradition médiévale de l'emprunt littéraire. En effet, les inscriptions rédigées pour des autels ou des églises, voire pour d'autres édifices, sont essentiellement des œuvres de " commande " et utilisent un stock limité de formules. Il ne faut pas y chercher l'originalité qui, de toute manière, n'est pas le trait dominant de la création littéraire carolingienne. On peut y reconnaître la prédominance de certaines expressions qui, même si elles sont empruntées à des textes antérieurs, épigraphiques ou non, apparaissent comme des "signatures " littéraires; ainsi, les inscriptions poitevines utilisent certaines expressions par ailleurs fréquentes dans l'ensemble des carmina d'Alcuin (vingt-deux fois in arce poli, seize fois decus ecclesiae, sept fois mentis vivacibus et precibus servet pour ne citer que les plus évidentes). Il faudrait là encore rendre ce repérage le plus systématique possible pour distinguer les véritables signatures ou marques d'auteur (que d'aucuns appellent " alcuinismes ") des lieux communs de la poésie carolingienne. En tous cas, si les inscriptions rédigées par Alcuin sont semblables, d'un établissement à l'autre, elles ne sauraient être considérées comme des répétitions au sens moderne du terme, étant destinées à des lieux distants de plusieurs centaines de kilomètres. On peut même se demander si par-delà la distance, l'usage de textes identiques ne parvenait pas à créer une véritable unité culturelle, que l'on retrouve très nettement, à l'échelle de l'empire, dans la réception épigraphique des carmina d'Alcuin.

\section{La réception épigraphique des carmina d'Alcuin}

L'édition de référence de Ernst Dümmler dans les Monumenta Germaniae Historica, bien pratique, donne l'impression (erronée) d'une grande cohérence des œuvres poétiques en général, et épigraphiques en particulier, d'Alcuin. La réalité est tout autre; un seul manuscrit - celui de Saint-Bertin, aujourd'hui disparu - regroupait la quasi-totalité des inscriptions rédigées par Alcuin à des dates et en des circonstances diverses. Elles ont, de ce fait, une diffusion différente selon les cas. L'une d'elles est insérée dans une œuvre hagiographique postérieure ${ }^{48}$, dont on possède encore cinq manuscrits médiévaux. Les inscriptions de Salzbourg sont connues par un manuscrit particulier, toujours conservé à Salzbourg et datant du $\mathrm{XI}^{\mathrm{e}}$ siècle ${ }^{49}$. Les inscriptions d'Arras, transmises par le manuscrit de SaintBertin, ont également fait l'objet de deux copies dans des florilèges locaux, plus tardifs. Deux compositions ${ }^{50}$ extraites de l'ensemble ont toutefois été copiées dans un manuscrit du IX ${ }^{\mathrm{e}}$ siècle conservé à Vienne ${ }^{51}$. Cette exception a sans doute un sens et il serait intéressant non seulement d'étudier

48. Vita Liudgeri, c. 17, éd. MGH SS 2, p. 409. M.-H. Jullen et F. Perelman, Clavis..., n AlC 61.86 .

49. Salzburg, Arch. S. Pet. AI

50. Haec domus alma Dei, et Pontificalis apex meritis, resp. ALC 61.89.1 et ALC 61.89.3 dans le répertoire de M.-H. JulLien et F. Perelman, Clavis...

51. Wien, Österreichische Nationalbibliothek, ms. 550. 
précisément la composition du manuscrit en question, afin d'en déterminer la logique, mais également de vérifier si ces deux textes n'ont pas connu une destinée épigraphique particulière.

Le regroupement géographique, sensible dans le manuscrit de SaintBertin et dans les florilèges locaux d'Arras, n'est pas forcément primordial. Ainsi, un manuscrit conservé à Vienne ${ }^{52}$, daté des environs de 802 et qui se trouvait à Salzbourg au temps d'Arno, regroupe successivement quatre inscriptions de Chelles, une attribuée à Cologne, deux de Fleury, deux de Tours et huit destinées à Saint-Pierre de Salzbourg, reprenant elles-mêmes des inscriptions de Poitiers, Saint-Amand et Arras. Une telle diversité pourrait être significative de la volonté d'Arno d'ancrer sa cité épiscopale dans la tradition des sanctuaires prestigieux et anciens du regnum Francorum, au moment où Salzbourg devient siège métropolitain pour favoriser l'extension vers l'est de ce même regnum. La reprise de textes préexistants, loin de relever d'un défaut d'imagination, aurait - si cette hypothèse est bonne - un sens politique fort.

Plusieurs des inscriptions rédigées par Alcuin ont vécu leur vie propre. Écrites pour une occasion spécifique, intégrées ou non dans une série pour un lieu précis, elles ont acquis très vite une véritable autonomie, s'affranchissant de la paternité d'Alcuin pour circuler seules et changeant même de genre littéraire. C'est ainsi que l'inscription rédigée pour le réfectoire des frères de Nouaillé (Christe Deus nostrae benedic), copiée sous l'autorité d'Alcuin mais de manière isolée et sans attribution de lieu dans un manuscrit du $\mathrm{X}^{\mathrm{e}}$ siècle conservé à Rome ${ }^{53}$, est transmise également comme hymne dans plusieurs manuscrits liturgiques des $\mathrm{IX}^{\mathrm{e}}-\mathrm{XI}{ }^{\mathrm{e}}$ siècles. Les titres qu'on peut y lire manifestent d'ailleurs cette fluctuation de genre littéraire : Epigramma de refectorio; Hymnus Benedictio mensae; Inscriptio ad refectorium; Versus ad mensam; Versus ante mensam ${ }^{54}$. Toutefois, les deux inscriptions les plus autonomes sont les épitaphes rédigées par Alcuin pour le pape Hadrien ${ }^{\text {er }}$ et pour lui-même.

L'épitaphe du pape Hadrien ${ }^{\mathrm{er}}$ est connue à la fois par une pierre sculptée, conservée à Saint-Pierre du Vatican à Rome (encastrée dans le mur de la façade occidentale, sous le portique) et par plusieurs manuscrits carolingiens ${ }^{55}$. Le tituusi circule de manière anonyme et son vers 17 cite le roi Charlemagne : Post patrem lacrimans Carolus haec carmina scripsi. Les Annales de Lorsch reprises par la Chronique de Moissac ne donnent pas le nom de l'auteur de cette inscription mais précisent que Charlemagne ordonna de la faire écrire, in Francia, en lettres d'or sur du marbre et d'envoyer à Rome pour orner la tombe du pape. Toutefois, l'attribution à Alcuin

52. Wien, Österreichische Nationalbibliothek, ms 808.

53. Roma, Biblioteca angelica, $\mathrm{ms} 1515, \mathrm{f}^{\circ} 31 \mathrm{r}^{\circ}$ (Versus Alcuini ante mensam).

54. Liste donnée par M.-H. Jullien et F. Perelman, Clavis..., n ${ }^{\circ}$ ALC 61.100.1.

55. Voir notamment la liste dressée par M.-H. JuLLIEN et F. PERELMAN, Clavis... Ces témoins manuscrits ont été étudiés par I. B. DE RossI, "L'inscription du tombeau d'Hadrien I... ", p. $481-483$. 
ne fait guère de doute lorsqu'on étudie précisément le discours, le vocabulaire et les formules utilisées. Deux florilèges poétiques, copiés au IX $\mathrm{eu}$ $\mathrm{X}^{\mathrm{e}}$ siècle ${ }^{56}$, reproduisent le texte sous le titre De Adriano papa, envisagé ici sous son aspect littéraire. En revanche, l'épitaphe d'Hadrien copiée à la fin d'un Liber pontificalis conservé à Paris semble faire office de notice biographique ${ }^{57}$. Elle peut être liée à une tradition manuscrite qui associe cette inscription à d'autres épitaphes pontificales, copiées directement à Rome sur leur support lapidaire. Ainsi, dans le manuscrit - aujourd'hui perdu de Saint-Pierre de Ratisbonne (exécuté sur ordre de Liutprand, évêque de Salzbourg), l'épitaphe d'Hadrien était suivie de 12 vers que Iohannes Baptista de Rossi a identifiés comme provenant de la tombe du pape Hormisdas ${ }^{58}$.

En ce qui concerne l'épitaphe d'Alcuin, la Vita Alcuini, en donnant le texte, précise qu'il l'avait lui-même rédigée et qu'elle avait été gravée sur une plaque de bronze fixée au mur dans l'église Saint-Martin qui reçut sa sépulture à Tours ${ }^{59}$. Cette inscription a aujourd'hui disparu mais le texte est connu par de très nombreux manuscrits sous différents titres et dans différents contextes ${ }^{60}$. Il est intégré dans des recueils de poètes carolingiens ou dans des compilations de nature " scolaire ", liées à l'enseignement de la grammaire et de l'orthographe. Il apparaît ainsi, associé à d'autres textes plus anciens, comme modèle de versification. En l'étudiant, tout lettré débutant l'apprenait et le gardait en mémoire, prêt à le réutiliser sans forcément l'avoir appris dans cette optique. Plus encore, très tôt, ce texte est devenu une véritable "formule " épigraphique. Dans ce qui pourrait être la plus ancienne copie connue, effectuée selon Donald A. Bullough peu après 805 à Bourges ${ }^{61}$ dans un manuscrit comprenant une collection de lois, de décrets et de capitulaires, et d'un ensemble « scolaire " (traité d'orthographe accompagné de quelques textes versifiés), l'épitaphe d'Alcuin n'est précédée d'aucun titre. En outre, aux deux endroits du texte où devrait apparaître le nom d'Alcuin, celui-ci a été remplacé par ill., marquant la transformation du statut du texte (figure 2) : de souvenir du grand grammairien, il est devenu formulaire épigraphique ${ }^{62}$. Ce phénomène se

56. Paris, Bibliothèque nationale de France, ms. lat. 9347, $\mathrm{f}^{\circ} 49 \mathrm{r}^{\circ}$ (De Adriano papa); on trouve également, au f $39 \mathrm{r}^{\circ}$, l'épitaphe d'Ethelbert d'York écrite par Alcuin, séparée de celle d'Hadrien par les épigrammes de Prosper; Paris, Bibliothèque nationale de France, ms. lat. 2773, $\mathrm{f}^{\circ} 23 \mathrm{v}^{\circ}$ (De Adriano papa).

57. Paris, Bibliothèque nationale de France, ms. lat. 16897, $\mathrm{f}^{\circ} 33 \mathrm{v}^{\circ}-34$ (Adrianus).

58. Voir L. DucheSNE, Le Liber pontificalis, 1, p. 274 et I. B. DE RossI, "L'inscription du tombeau d'Hadrien I... ", p. 491-492.

59. Super cuius tumulum positus est, sicut ipse iusserat, titulus, quem ipse vivens dictaverat, lamina scriptus in aerea parietique insertus. Éd. MGH SS, XV, p. 197.

60. Voir notamment la liste dressée par M.-H. JuLLIEN et F. PERELMAN, Clavis... Plusieurs témoins, parmi les plus anciens, ont été étudiés par D. Bullough, Alcuin. Achievement and Reputation..., Additionnal Note II (The early manuscript evidence for Alcuin's epitaph), p. 122.

61. Paris, Bibliothèque nationale de France, ms. lat. $4629, \mathrm{f}^{\circ} 55 \mathrm{v}^{\circ}$.

62. Après le qui obiit in pace, on observe également un blanc, destiné à noter la date de la mort du personnage concerné par l'utilisation de ce «formulaire ». Les inscriptions 


\section{Figure 2}

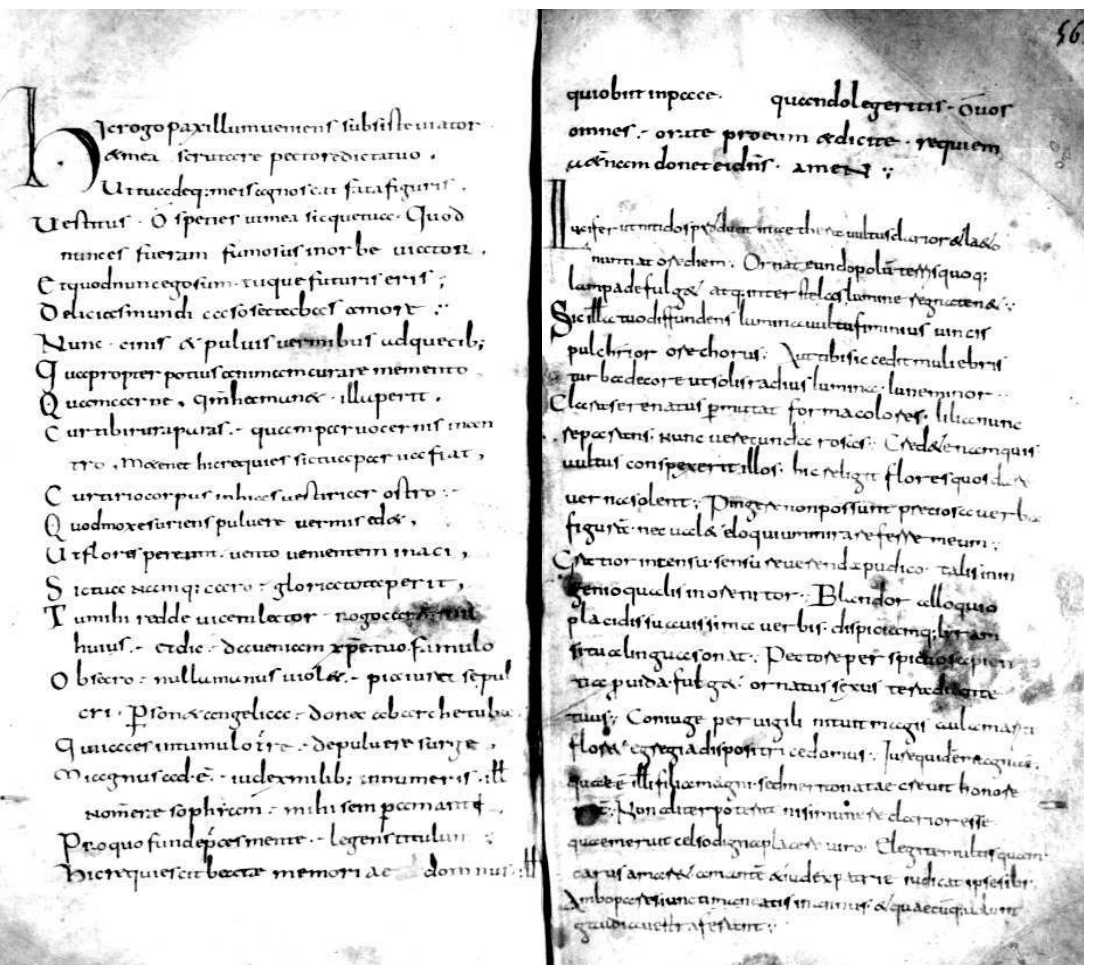

retrouve dans plusieurs manuscrits, dont un autre conservé à Paris ${ }^{63}$ ou celui de Munich ${ }^{64}$ qui, signalé par D. Bullough, développe le texte sous le titre Epitaphium super sepulchrum avec deux blancs à la place du nom d'Alcuin.

La postérité de cette inscription dans les compositions épigraphiques littéraires est importante; elle a été source d'inspiration pour la rédaction des épitaphes d'Angilbert ${ }^{65}$, de Salomon ${ }^{66}$, de Wolfart ${ }^{67}$ ou de Walafrid

carolingiennes conservées à Bourges (éd. F. JENN, O. RUFFIER, "Les plates-tombes inscrites..." ) ne portent cependant pas trace d'un quelconque emprunt à cette inscription. En revanche, l'atelier situé vraisemblablement près de Saint-Oustrille semble avoir travaillé en composant les épitaphes grâce à un formulaire propre. Voir, en attendant un travail plus complet, C. TREFFORT, "Corps individuel, corps social... ".

63. Paris, Bibliothèque nationale de France, ms. $4841, \mathrm{f}^{\circ} 33$, où la formule Alchuine nomen erat est remplacée par Mihi illud nomen erat et où la dernière partie, avec le jour de la mort et la formule de prière est omise. Voir I. B. DE Rossi, Inscriptiones christianae..., vol. II, 1, p. 277.

64. München, Staatsbibliothek, ms. Clm 18628, $\mathrm{f}^{\circ} 93 \mathrm{v}^{\circ}$.

65. Reconstituée par L. TrauBE, Karolingische Dichtungen, Berlin, 1888, p. 55, cité par L. WaLlach, "The Epitaph of Alcuin... ", p. 371, n. 14.

66. MGH Poet. 2, p. 656.

67. MGH Poet. 2, p. 410. 
Strabon par lui-même ${ }^{68}$. On garde également des témoins lapidaires de la réception de ce texte, notamment dans l'épitaphe de l'archidiacre Pacificus de Vérone, mort en $846^{69}$, dont l'inscription funéraire, peut-être réalisée au $\mathrm{Xl}^{\mathrm{e}}$ siècle ${ }^{70}$ et encore conservée dans la cathédrale de Vérone, réutilise tous les vers de l'épitaphe d'Alcuin, exceptées quelques lignes ${ }^{71}$ :

\begin{tabular}{|c|c|}
\hline Inscription de Pacificus de Vérone & Épitaphe d'Alcuin \\
\hline \multicolumn{2}{|l|}{ 1- Hic rogo pauxillum subsiste viator (Alc. 1) } \\
\hline \multicolumn{2}{|l|}{ 2- Et mea scrutare pectore dicta tuo (Alc. 2) } \\
\hline & Ut tua deque meis agnoscas fata figuris (Alc. 3) \\
\hline & Vertitur o species, ut mea, sicque tua (Alc. 4) \\
\hline \multicolumn{2}{|c|}{ 3- Quod nunc es fueram famosus in orbe viator (Alc. 5) } \\
\hline \multicolumn{2}{|l|}{ 4- Et quod nunc ego sum tuque futurus eris (Alc. 6) } \\
\hline \multicolumn{2}{|l|}{ 5- Delicias mundi pravo sectabar amore (Alc. 7) } \\
\hline \multicolumn{2}{|l|}{ 6- Nunc cinis et pulvis vermibus atque cibus (Alc. 8) } \\
\hline \multicolumn{2}{|l|}{ 7- Quapropter potius animam curare memento (Alc. 9) } \\
\hline \multicolumn{2}{|c|}{ 8- Quam carnem quoniam haec manet illa perit (Alc. 10) } \\
\hline \multicolumn{2}{|c|}{ 9- Cur tibi plura paras quam parvo cernis in antro (Alc. 11) } \\
\hline \multicolumn{2}{|l|}{ 10- Me tenet hic requies sic tua parva fiet (Alc. 12) } \\
\hline \multicolumn{2}{|l|}{ 11- Ut flores pereunt vento veniente minaci (Alc. 15) } \\
\hline \multicolumn{2}{|l|}{ 12- Sic tua namque caro gloria tota perit (Alc. 16) } \\
\hline \multicolumn{2}{|c|}{ 13- Tu mihi redde vicem lector rogo carminis huius (Alc. 17) } \\
\hline \multicolumn{2}{|l|}{ 14- Et dic Da veniam, Christe, tuo famulo (Alc. 18) } \\
\hline 15- Pacificus Salomon mihi nomen atque Ireneus & $\begin{array}{l}\text { Alchuine nomen erat sophiam mihi semper } \\
\text { amanti (Alc. 25) }\end{array}$ \\
\hline \multicolumn{2}{|l|}{ 16- Pro quo funde preces mente legens titulum (Alc. 24) } \\
\hline \multicolumn{2}{|c|}{ 17- Obsecro nulla manus violet pia iura sepulcri (Alc. 19) } \\
\hline \multicolumn{2}{|c|}{ 18- Personet angelica donec ab arce tuba (Alc. 20) } \\
\hline \multicolumn{2}{|c|}{ 19- Quid iaces in tumulo terrae de pulvere surge (Alc. 21) } \\
\hline \multicolumn{2}{|l|}{ 20- Magnus adest iudex milibus innumeris (Alc. 22) } \\
\hline \multicolumn{2}{|l|}{ 21- Tolle hic segnitiem pone fastidia mentis } \\
\hline \multicolumn{2}{|l|}{ 22- Crede mihi frater doctior hinc redies } \\
\hline 23-Anno dominice incarnationis DCCCXLVI indicte $X$ & \\
\hline
\end{tabular}

Les emprunts sont rarement aussi massifs ou aussi évidents et il faut habituellement scruter très attentivement les témoins lapidaires pour y reconnaître des formules parentes de celles utilisées par Alcuin. Un vers de l'épitaphe écrite par ce dernier vers 793 pour Gislebert, évêque de Noyon et abbé de Saint-Amand ${ }^{72}$ enterré dans l'église Saint-Pierre de ce monastère, est identique à celui qu'on trouve sur l'épitaphe lapidaire de l'évêque de Würzburg, Megingoz, mort en 794 : terram terra tenet spiritus astra petit ${ }^{73}$. La

68. MGH Poet. 2, p. 424.

69. MGH Poet. 2, p. 655-656.

70. Si l'on suit l'argumentation de C. La Rocca, Pacifico di Verona..., p. 159-172.

71. Cette parenté avait été étudiée dès 1936 par H. HEngst, Totenklage und Nachruf..., p. 19-20. Le tableau qui suit ne tient pas compte des variantes minimes perceptibles dans l'épitaphe de Pacificus, qui peuvent provenir de la tradition manuscrite de celle d'Alcuin dans la version utilisée, inconnue à ce jour.

72. Alc., Carm. 88, 1, v. 2, éd. MGH Poet. 1, p. 305.

73. Die deutschen Inschriften, $27, \mathrm{n}^{\circ} 1$, p. 3-4. 
deuxième partie de ce vers, qui reprend un passage de l'épitaphe de Grégoire le Grand ${ }^{74}$, se trouvait déjà dans la Vita de saint Willibrord et dans le poème sur l'église d'York par Alcuin, mais en début de vers ${ }^{75}$. Dans une autre épitaphe de Megingoz, transmise par la tradition manuscrite, un vers se rapproche également de la poésie d'Alcuin ${ }^{76}$. L'incertitude de datation et la proximité chronologique de ces différents textes posent le problème du rapport exact entre eux. Les épitaphes de Megingoz sont-elles dépendantes de l'œuvre d'Alcuin? Puisent-elles à la même source? Le phénomène d'emprunt pourrait-il aller dans l'autre sens, Alcuin s'étant inspiré de ces inscriptions funéraires? Il semble impossible, dans le cas présent, de trancher définitivement.

On peut toutefois évoquer, pour terminer, un dernier exemple qui ne laisse aucun doute sur l'emprunt alcuinien du rédacteur malheureusement anonyme; il s'agit de l'épitaphe lapidaire d'un abbé angevin nommé Ato, découverte au XIX ${ }^{\mathrm{e}}$ siècle (figure 3 ) et aujourd'hui conservée au Musée des antiquités à Angers ${ }^{77}$. L'inscription, sculptée sur une belle dalle d'ardoise de plus de 2 mètres de long et lourde de plusieurs centaines de kilos, datée de 835 comme nous l'apprend le cartouche latéral, puise dans le poème de l'Église d'York ${ }^{78}$ avec une virtuosité remarquable :

\begin{tabular}{|l|l|}
\hline Épitaphe d'Ato à Angers (835) & Poème d'Alcuin sur l'Église d'York \\
\hline 1- Mausoleo felix recubat Ato abba in isto & Nobilium coram saeclo radice parentum (v. 1251) \\
\hline 2- Ortus nobilium sat de radice parentum & $\begin{array}{l}\text { Sed Domino coram meritis praeclarior almis } \\
\text { (v. 1252) }\end{array}$ \\
\hline 3-Sed Domino coram meritis nobilior almis & Qui fuit a puero signis insignis apertis (v. 647) \\
\hline 4- Nam fuit a puero semper pius atque modestus & Moribus egregius Christi mandata secutus (v. 268) \\
\hline 5- Moribus egregius Christi mandata secutus & Hostibus horribilis, cunctis iocundus amicis (v. 272) \\
\hline 6- Omnibus affabilis cunctis iocundus amicis & $\begin{array}{l}\text { Transit in/ad aetheream laetus feliciter aulam } \\
\text { (v. 875/1568) }\end{array}$ \\
\hline 7- Iuit ad aetheream laetus feliciter aulam & \\
\hline 8- Pro quo qui legitis fundite posco preces. & \\
\hline
\end{tabular}

Le doute est bien sûr toujours permis, notamment lorsqu'on regarde le vers 4 , qui n'est guère concluant; on trouverait sans doute d'autres œuvres utilisant les mêmes formules. Toutefois, il est difficile de croire qu'une telle

74. I. B. DE RossI, Inscriptiones christianae..., vol. II, $1, \mathrm{n}^{\circ} \mathrm{V}, 1, \mathrm{p} .52, \mathrm{n}^{\circ} \mathrm{VII}, 3$, p. $78, \mathrm{n}^{\circ} \mathrm{VIII}$, 73, p. 112, n XIX, 38, p. 209.

75. Alcuin, Vita Willibrordi, II, 28, 4, éd. MGH Poet. 1, p. 216 : Spiritus astra petit meritis vivacibus alta; Poème sur l'Eglise d'York, v. 739, éd. ibid., p. 186 : Spiritus astra petit sancti terrena relinquens. Voir L. WALLACH, "Alcuin's Epitaph of Hadrian I... ", p. 143.

76. Cf. M. GARRISON, "Alcuin, carmen IX and Hrabanus... », p. 69.

77. Angers, Musée des Antiquités (réserves Notre-Dame), n inv. GF 24. Découverte dans les fouilles de la place du Ralliement, cette inscription a été publiée pour la première fois par V. Godard-Faultrier, "Épitaphe de l'abbé Ato ", qui avait remarqué le caractère métrique du texte, sans soupçonner les emprunts à Alcuin. Une étude complète de cette épitaphe est sous presse (C. Treffort, "Un témoin de la vie politique..." "). Je tiens à remercier à cette occasion Catherine Lesseur, conservateur, pour son accueil, sa disponibilité et son efficacité.

78. Éd. P. Godman, Alcuin, The Bishops, Kings... 


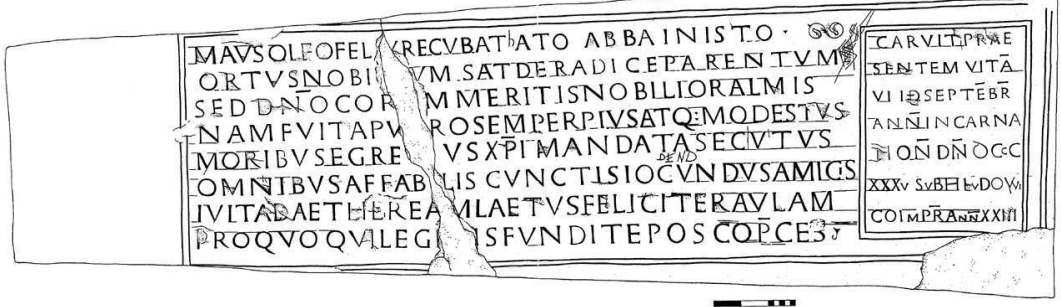

coïncidence relève seulement du hasard. Les vers 3,5 et 7 ne présentent que de très légères variantes par rapport au poème d'Alcuin dans la version qui nous est parvenue. En outre, Angers ne se trouve guère qu'à une centaine de kilomètres de Tours (comme Poitiers ou Fleury-sur-Loire); une trentaine d'années seulement séparent la mort d'Alcuin de celle d'Ato, qui peut l'avoir connu personnellement et même avoir bénéficié de son enseignement à Saint-Martin. Il semble donc plus probable que celui qui a composé cette inscription - peut-être Ato lui-même - connaissait parfaitement le poème d'Alcuin sur l'Église d'York et l'a utilisé sciemment. Or, la tradition manuscrite de ce texte est actuellement réduite à deux manuscrits champenois, dont un seul, datant du XII ${ }^{\mathrm{e}}$ siècle, est conservé ${ }^{79}$. L'épitaphe d'Ato représente pour l'instant le plus ancien témoin d'une tradition indirecte de ce texte, d'autant plus précieux qu'il est bien daté, et les variantes relevées dans les vers 3,5 et 7 pourraient même correspondre à une tradition antérieure à celle du manuscrit conservé.

Il y a bien évidemment, dans la pratique épigraphique, différents niveaux d'inscriptions, de la plus courte à la plus longue, de la plus simple à la plus sophistiquée. Nous n'avons évoqué ici que quelques exemples particulièrement significatifs pour montrer l'importance des liens entre la composition poétique et la pratique épigraphique jusque dans sa dimension matérielle. Les diverses réflexions fondées sur un exemple unique ne forment certes pas un dogme; elles sont toutefois suffisantes pour prouver la réalité - sinon la fréquence - non seulement des emprunts d'Alcuin aux textes épigraphiques antérieurs, mais également de la réception de ses propres carmina, épigraphiques ou non, dans les inscriptions postérieures, quel qu'en soit le mode de transmission. Si la composition d'inscriptions versifiées, notamment par l'usage du procédé du centon, relève, à n'en point douter, d'un haut niveau de culture écrite, elle n'est ni cantonnée à la pratique manuscrite, ni limitée aux rois, aux princes et aux grands ecclésiastiques. En lisant avec attention les pierres parfois monumentales que nous ont léguées inconnus et anonymes de l'époque carolingienne, on

79. Reims, Bibliothèque municipale, ms. 426. Voir Fr. DolBEAU, " La tradition textuelle du poème d'Alcuin..." ". 
pourra reprendre de manière différente le problème de la place des inscriptions littéraires et réelles dans la culture carolingienne.

\section{RESUME}

Souvent ignorées des chercheurs, les inscriptions font pourtant partie de la culture écrite carolingienne, comme le prouve parfaitement l'œuvre d'Alcuin. Celui-ci a en effet rédigé de nombreux textes épigraphiques, en particulier l'épitaphe du pape Hadrien I ${ }^{\text {er }}$ et la sienne, célèbres dans le monde carolingien. Il a également utilisé des formules provenant d'inscriptions anciennes, notamment d'épitaphes pontificales transmises par les sylloges manuscrites. Enfin, ses œuvres poétiques ont été sources d'inspiration pour les rédacteurs d'inscriptions, comme en témoignent certains de ses vers gravés dans la pierre longtemps après sa mort. L'étude de la place des inscriptions dans l'œuvre d'Alcuin et, parallèlement, celle de la place d'Alcuin dans la production épigraphique permettent de reconsidérer la culture écrite carolingienne sous un nouvel angle, désormais indispensable pour en comprendre la richesse.

\section{ABSTRACT}

Historians are often unaware of inscriptions; however, epigraphical evidence belongs to Carolingian literacy, as Alcuin's textual production proves it. Indeed, Alcuin wrote many epigraphical poems and two of them (the pope Hadrian I's epitaph and his own one) where famous in the Carolingian world. When he composed his Poem on York or his carmina, he used as well expressions and forms coming from ancient inscriptions, especially from pontifical epitaphs he knew through manuscripts and sylloges. At last, his poetic production has been used by inscription's writers, as we can see with some Alcuin's verses carved on stone many years after his death. With Alcuin's example, this study describes the link existing between poetic literature and epigraphical traditions or productions, showing inscriptions as an essential source for studying and understanding Carolingian literacy, culture and history. 
